\title{
METRIC SEMANTICS FOR CONCURRENCY
}

\section{J. W. DE BAKKER and J.J. CH. MEYER}

Centre for Mathematics and Computer Science, Kruislaan 413, NL-1098 SJ Amsterdam,

Free University of Amsterdam,

The Netherlands
Free University of Amsterdam, De Boelelaan 1081, NL-1081 HV Amsterdam, The Netherlands

Dedicated to Peter Naur on the occasion of his 60th birthday

\begin{abstract}
.
An overview is given of work we have done in recent years on the semantics of concurrency, concentrating on semantic models built on metric structures. Three contrasting themes are discussed, viz. (i) uniform or schematic versus nonuniform or interpreted languages; (ii) operational versus denotational semantics, and (iii) linear time versus branching time models. The operational models are based on Plotkin's transition systems. Language constructs which receive particular attention are recursion and merge, synchronization and global nondeterminacy, process creation, and communication with value passing. Various semantic equivalence results are established. Both in the definitions and in the derivation of these equivalences, essential use is made of Banach's theorem for contracting functions.
\end{abstract}

1985 Mathematics Subject Classification: 68Q55, 68Q10.

1987 Computing Reviews Categories: D.1.3, D.3.1, D.3.3, F.1.2, F.3.2.

Keywords: concurrency, operational semantics, denotational semantics, transition systems, process creation, synchronization, metric spaces, domain equations, contracting functions, global nondeterminacy.

\section{Introduction.}

We present an expository account of work we have been pursuing in recent years on the semantics of concurrency, concentrating on those models which are built on structures from metric topology. We shall exhibit semantic definitions for a variety of programming notions relating to concurrency, viz. recursion with merge (parallel execution in the interleaving sense), synchronization and global nondeterminacy, process creation, and communication with value passing. We hope to demonstrate the power of metric methods, both in the semantic definitions themselves and in the establishment of particularly succint derivations of equivalence results between operational and denotational semantic models.

Received October 1987. Revised April 1988. 
Three contrasting themes will recur in our considerations (cf. [7] for a more elaborate treatment). First, there is the familiar distinction between operational and denotational semantics. The former will always be based on transition systems which are variations on the elegant systems of Hennessy and Plotkin ([16], [25], [26]). The latter will throughout be defined compositionally, with (unique) fixed points to deal with recursion. Such fixed points exist on the basis of Banach's theorem for contracting functions. In fact, this theorem is absolutely pervasive in our technical considerations: a good deal of our definitions and theorems ultimately rely on it. Second, we shall contrast uniform and nonuniform languages. The former are schematic in the sense that their elementary actions are uninterpreted, and the meanings rendered by our definitions involve entities with a strong flavour of formal language theory. More specifically, sets of (possibly infinite) words or tree-like objects are delivered. Nonuniform languages have interpreted elementary actions. They include notions such as (individual) variables, assignments, states and state transforming functions. As we shall demonstrate, it requires additional tools to set up a framework in which one may merge such functions. Third, we shall be concerned with both linear time (LT) and branching time (BT) models. Typical examples are sets of words versus trees (with some further properties not stated here) over some alphabet $A$. In the former, moments of choice are abstracted away which are present in the latter. We recall the classical example of the LT set $\{a b, a c\}$ versus the two different trees in BT:
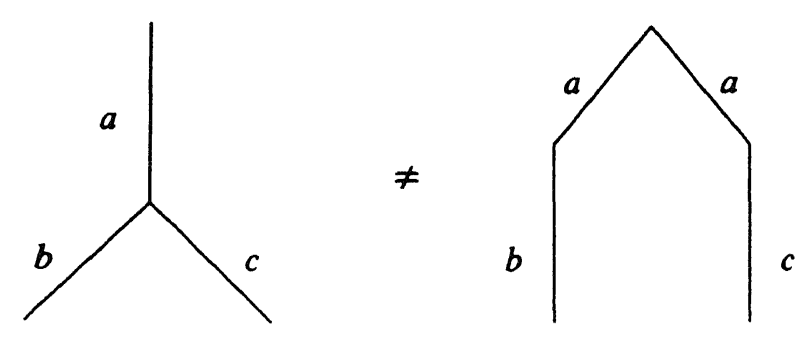

The genealogy of the work described in the present paper is as follows: Ancestors are Nivat's work on metric techniques in semantics ([23]) and Plotkin's work on resumptions in power domains ([24]). In [11] we described a general method to solve domain equations using metric techniques. [12] is an example of a specific semantic application. A substantial improvement on [11] is given in [4] where the scope of the method in [11] was clarified and, even better, considerably generalized. A comparison of LT and BT models for recursion with merge was first made in [6]. In [10], [7] a systematic comparison of operational 
and denotational models was developed, both for recursion and merge, for synchronization with (forms of) nondeterminacy, and for nonuniform languages. Somewhat simultaneously we have devoted a number of papers to the design of semantic models for the parallel object oriented language POOL $([2,3,1])$, dealing, besides with various other notions, with process creation. An essential step on the way to substantial simplification of the sometimes quite elaborate arguments in [10], [1] was performed in [18]. Here the full power of the unique fixed point argument, not only in defining but also in comparing semantic models, was first exploited.

In parallel to the metrically based semantic studies, we have also continued to work with models based on partial orders, were it only to relate order-theoretic models to metric ones. In addition, for the metric models as we use them, the requirement that all sets considered be closed is vital, and the metric theory fails when phenomena inducing nonclosed sets are encountered. Examples of comparative studies, in particular relating to the "elemental" combination of recursion with merge, are [8], [9]. An extensive application of order-theoretic tools, specifically to deal with fair merge (the result of which is in general nonclosed) is described in [19]. Another language notion which is not directly amenable to metric techniques is that of hiding (cf. [20]). Finally, we mention [21] where an order-theoretic counterpart of the topological notion of compactness is studied.

More in general, the relationship between the metric and order-theoretic domain theory is a topic of much current research. A representative reference is [27].

We are at present investigating further applications of the metric method in semantics. Two prime examples are uniform (or "logicless") versions of logic programming, and more advanced concepts in object-oriented programming.

\section{Mathematical preliminaries.}

\subsection{Notation.}

The phrase "let $(x \in) X$ be such that ..." introduces a set $X$ with variable $x$ ranging over $X$ such that .... For $X$ a set, $\mathscr{P}(X)$ denotes the collection of all subsets of $X$, and $\mathscr{P}_{\pi}(X)$ is the collection of all subsets of $X$ which have property $\pi$. The notation $f: X \rightarrow Y$ expresses that $f$ is a function with domain $X$ and range $Y$. We use the notation $f\{y / x\}$, with $x \in X$ and $y \in Y$, for a variant of $f$, i.e. for the function which is defined by

$$
f\{y / x\}\left(x^{\prime}\right)= \begin{cases}y & \text { if } x=x^{\prime} \\ f\left(x^{\prime}\right) & \text { otherwise }\end{cases}
$$

If $f: X \rightarrow X$ and $f(x)=x$, we call $x$ a fixed point of $f$. 


\subsection{Metric spaces.}

From standard topology (e.g. [14], [15]) we assume known the notion of (ultra)metric space (M, d) with distance or (ultra)metric $\mathbf{d}$. We use the notions of closed subset $X$ of $(\mathbf{M}, \mathbf{d})$, of continuous mapping $\left(\mathbf{M}_{1}, \mathbf{d}_{1}\right) \rightarrow\left(\mathbf{M}_{2}, \mathbf{d}_{2}\right)$, of completeness of a metric space, and of isometry $(\cong)$ between metric spaces $\left(\mathbf{M}_{1}, \mathbf{d}_{1}\right)$ and $\left(\mathbf{M}_{2}, \mathbf{d}_{2}\right)$. A mapping $f:\left(\mathbf{M}_{1}, \mathbf{d}_{1}\right) \rightarrow\left(\mathbf{M}_{2}, \mathbf{d}_{2}\right)$ is called contracting whenever, for all $x, y \in \mathbf{M}_{1}$, we have $\mathbf{d}_{2}(f(x), f(y)) \leqq \alpha \cdot \mathbf{d}_{1}(x, y)$, with $0 \leqq \alpha<1$. If the same condition holds with $\alpha=1$, we call $f$ non distance increasing (ndi). Clearly, a contracting or ndi mapping is continuous. A central role is played below by

Proposition 2.1 (Banach). Let $f:(\mathbf{M}, \mathbf{d}) \rightarrow(\mathbf{M}, \mathbf{d})$ be contracting, and let $(\mathbf{M}, \mathbf{d})$ be complete. Then $f$ has a unique fixed point $x_{0}$ and, for any $y, x_{0}=\lim _{i} f^{i}(y)$, where $f^{0}=\lambda x \cdot x, f^{i+1}=f \circ f^{i}$.

\subsection{Metric spaces of (sets of) words.}

Let $A$ be a finite alphabet, let $A^{*}\left(A^{\omega}\right)$ denote the collection of all finite (infinite) words over $A$, and let $A^{\infty}={ }^{d f} \cdot A^{*} \cup A^{\omega}$. Let $\varepsilon$ denote the empty word. For each $u \in A^{\infty}, u(n)$ is the prefix of $u$ of length $n$, if this exists, and $u(n)=u$, otherwise. We define a metric $\mathbf{d}$ on $A^{\infty}$ by putting $\mathbf{d}(u, v)=2^{-n}$, where $n=\sup \{k \mid u(k)=v(k)\}$. Thus, $\mathbf{d}(u, v)=2^{-\infty}=0$ if $u=v$. We have

Proposition 2.2. $\left(A^{\infty}, \mathrm{d}\right)$ is a complete ultrametric space.

In $\left(A^{\infty}, \mathrm{d}\right)$ we have, e.g., that $\lim _{n} u(n)=u$. A typical closed subset of $\left(A^{\infty}, \mathrm{d}\right)$ is $a^{*} \cdot b \cup\left\{a^{\omega}\right\}$, whereas $a^{*} \cdot b$ is not closed. Let $Q=\mathscr{P}_{n c}\left(A^{\infty}\right)$ denote the collection of all nonempty closed subsets of $A^{\infty}$. Let, for $X \in Q, X(n)=\{u(n) \mid u \in X\}$. We define a metric $\mathbf{d}$ on $Q$ by putting $\mathrm{d}(X, Y)=2^{-n}$, where $n=\sup \{k \mid X(k)=Y(k)\}$. For example, $\mathbf{d}(\{a b c, e f\},\{a b c d, e f g\})=2^{-2}$. We have

Proposition 2.3. $(Q, \mathbf{d})$ is a complete ultrametric space.

\subsection{Domain equations and resumptions.}

We briefly recall the notion of a (metric) domain equation. The general form of such an equation is

$$
P \cong \mathscr{F}(P)
$$

or, more precisely, $\left(\boldsymbol{P}, \mathbf{d}_{\boldsymbol{P}}\right)=\mathscr{F}\left(\left(\boldsymbol{P}, \mathbf{d}_{\mathbf{P}}\right)\right)$, where the mapping $\mathscr{F}$ (technically a functor from the category of complete metric spaces to itself, but we do not have to be aware of this) is built up as follows: $\mathscr{F}$ is either a constant (delivering some complete $\left.\left(A, \mathbf{d}_{A}\right)\right)$, a transformation $i d_{\alpha}$ which maps $(\mathbf{M}, \mathbf{d})$ to $(\mathbf{M}, \alpha \cdot \mathbf{d})$ 
for some real $\alpha$, or composed from already given components by operations such as cartesian product, disjoint union, (restricted) function spaces, or the "closed subset of" mapping. We have no room to discuss details which are described at length in [11] or [4] (see also [13] for the connection between such $P$ and spaces obtained through bisimulation from synchronization trees as, e.g., in [22]). It is sufficient to know that isometries such as

$$
\begin{aligned}
& P \cong\left\{p_{0}\right\} \cup\left(A \times i d_{1 / 2}(P)\right) \\
& P \cong\left\{p_{0}\right\} \cup \mathscr{P}_{\text {closed }}\left(A \times i d_{1 / 2}(P)\right) \\
& P \cong\left\{p_{0}\right\} \cup\left(A \rightarrow \mathscr{P}_{\text {closed }}\left(B \times i d_{1 / 2}(P)\right)\right)
\end{aligned}
$$

all have well-defined solutions as complete metric spaces. For examples, $P$ as in (2.2) consists of $p_{0}$, all finite sequences $\left.\left\langle a_{1}\left\langle a_{2}, \ldots,\left\langle a_{n}, p_{0}\right\rangle \ldots\right\rangle\right\rangle, n\right\rangle 0$, and all infinite sequences $\left\langle a_{1},\left\langle a_{2}, \ldots,\left\langle a_{n}, \ldots\right\rangle \ldots\right\rangle\right\rangle$. Elements of each $P$ are either finite (and then equal to $p_{0}$ or in some $P_{n+1}=\mathscr{F}\left(P_{n}\right)$ ), or infinite and then satisfy $p=\lim _{n} p_{n}$, with $p_{n} \in P_{n}$. Occurrences of $P$ in terms $\ldots \times P$ on the right-hand side of these equations justify the terminology of resumptions: For example, for $p \in P$ with $P$ as in (2.4), $p\left(\neq p_{0}\right)$ is a function which, when supplied with argument $a \in A$ turns itself into, among other things, some $\left\langle b, p^{\prime}\right\rangle$. In later applications we shall read this with the connotation: process $p$ maps $a$ to $b$ and then turns itself into process $p^{\prime}$ as resumption.

For subsequent purposes, we note that, if the constant spaces $\left(A, \mathbf{d}_{A}\right),\left(B, \mathbf{d}_{B}\right), \ldots$ are assumed to be ultrametric, then the solutions $P$ (as in (2.2) to (2.4)) are also ultrametric.

Example: Elements from $P$ as in (2.3) are, e.g., $\left\{\left\langle a,\left\{\left\langle b, p_{0}\right\rangle,\left\langle c, p_{0}\right\rangle\right\}\right\rangle\right\}$ and $\left\{\left\langle a,\left\{\left\langle b, p_{0}\right\rangle\right\}\right\rangle,\left\langle a,\left\{\left\langle c, p_{0}\right\rangle\right\}\right\rangle\right\}$. These may be pictorially represented by the trees from section 1 . No such distinction is present in the set $Q$, where both objects are represented by the set $\{a b, a c\}$.

\section{Recursion and merge.}

The first language we consider is a simple extension of the traditional (uniform) sequential languages, obtained by adding the programming construct of parallel execution or merge $s_{1} \| s_{2}$ of the two statements $s_{1}$ and $s_{2}$. By a traditional (uniform) language we mean here a language which has (uninterpreted) elementary actions taken from some alphabet $A$, sequential composition, nondeterministic choice and recursion. It is well-known that these four concepts put together in the customary way - the exact syntax follows in a moment - yield the expressive power of context-free languages, here taken in the general sense of languages over finite and infinite words over $A$. Thus, we may rephrase the object 
of study in the present section as infinitary context-free languages extended witn merge or shuffle, where the latter notion is the standard operation of language theory. This combination of (basic notions with) recursion and merge was first studied in [6] (denotational LT and BT models) and [10,7] (operational vs. denotational LT models). The presentation below essentially follows [18], though our returning here to the format of simultaneous recursion - rather than employing possibly nested $\mu$-constructs - allows a considerably more concise treatment.

We build the syntax starting from

- a (not necessarily finite) alphabet $A$, with elements $a, b, c, \ldots$

- a set $\mathscr{P} v a r$ of procedure variables $x_{1}, x_{2}, \ldots$ It will be convenient to assume that each program uses exactly the procedure variables in the initial fragment $\mathscr{X}=\left\{x_{1}, \ldots, x_{n}\right\}$ of $\mathscr{P}_{\text {var }}$, for some $n \geqq 0$.

We start with

Definition 3.1 (Syntax).

a (statements). The class $(s \in) \mathscr{L}_{1}$ of statements is given by

$$
s::=a|x| s_{1} ; s_{2}\left|s_{1} \cup s_{2}\right| s_{1} \| s_{2} \text {, with } x \in \mathscr{X}
$$

b (guarded statements). The class $(g \in) \mathscr{L}_{1}^{g}$ of guarded statements is given by

$$
g::=a|g ; s| g_{1} \cup g_{2} \mid g_{1} \| g_{2}
$$

c (declarations). The class $(D \in) \mathscr{D}_{\text {ect }}{ }_{1}$ of declarations consists of $n$-tuples $D \equiv x_{1} \Leftarrow g_{1}, \ldots, x_{n} \Leftarrow g_{n}$ or $\left\langle x_{i} \Leftarrow g_{i}\right\rangle_{i}$, for short, with $x_{i} \in \mathscr{X}$ and $g_{i} \in \mathscr{L}_{1}^{g}$.

d (programs). The class $(t \in) \mathscr{P P}_{\mathrm{rog}_{1}}$ of programs consists of pairs $t \equiv\langle D \mid s\rangle$, with $D \in \mathscr{D}_{e x \ell_{1}}$ and $s \in \mathscr{L}_{1}$.

\section{EXAMPLES.}

1. <

$$
\begin{aligned}
& x_{1} \Leftarrow a ; x_{2} \cup b ; x_{3}, \\
& x_{2} \Leftarrow b \cup b ; x_{1} \cup a ; x_{3} ; x_{3}, \\
& x_{3} \Leftarrow a \cup a ; x_{1} \cup b ; x_{2} ; x_{2}, \\
& \left|x_{1}\right\rangle
\end{aligned}
$$

2. $\langle x \Leftarrow a ;(b \| x) \mid(c \| x)\rangle$

\section{REMARKS.}

1. We find it convenient not to worry about the ambiguity in the syntax for $\mathscr{L}_{1}$ (and the other languages that we shall define in the sequel). If required, the reader may add parentheses around the composite constructs, or assign priorities to the operators. 
2. All $g_{i}$ occurring in a declaration $D \equiv\left\langle x_{i} \Leftarrow g_{i}\right\rangle_{i}$ are required bo be guarded, i.e. occurrences of $x \in \mathscr{X}$ in $g_{i}$ are to be preceded by some $g$ (which, by clause $b$, has to start with an elementary action). This requirement corresponds to the usual Greibach condition in formal language theory.

3. We have adopted the simultaneous declaration format for recursion rather than the $\mu$-formalism which features constructs such as, for example, $c \| \mu x\left[a ;\left(\mu y\left[b_{1} ; y ; b_{2} \cup b_{3}\right] \| x\right)\right]$. As remarked already, the avoidance of (nested) $\mu$-constructs allows for a simpler derivation of the main semantic equivalence result to follow.

We proceed with the definitions leading up to the operational semantics for $s \in \mathscr{L}_{1}$ and $t \in \mathscr{P}_{\text {rog }}$. It is convenient to extend $\mathscr{L}_{1}$ with a special "empty statement" $E$ which performs no action (it will obtain $\{\varepsilon\}$ as its meaning). We put $\mathscr{L}_{1}^{\prime}=\mathscr{L}_{1} \cup\{E\}$. The operational semantics is based on transitions (following the operational semantics techniques of Structured Operational Semantics, cf. $[16,25,26])$. Here, transitions are four-tuples in $\mathscr{L}_{1} \times A \times \mathscr{D}_{e c t_{1}} \times \mathscr{L}_{1}^{\prime}$, written in the notation

$$
s \stackrel{a}{\rightarrow} s^{\prime}
$$

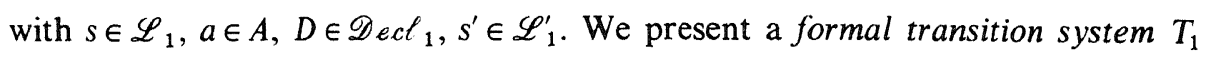
which consists of axioms and rules. Transitions which are given as axioms hold by definition. Moreover, a transition which is the consequence of a rule holds in $T_{1}$ whenever it can be established that, according to $T_{1}$, its premise holds (or, in later sections, its premises hold). We shall employ below the following notational variants of the format of rules: We use

$$
\frac{1 \rightarrow 2 \mid 3}{4 \rightarrow 5 \mid 6}
$$

to abbreviate

$$
\frac{1 \rightarrow 2}{4 \rightarrow 5} \text { and } \frac{1 \rightarrow 3}{4 \rightarrow 6}
$$

Also,

$$
\begin{gathered}
\frac{1 \rightarrow 1^{\prime}}{2 \rightarrow 2^{\prime}} \\
\quad \cdots \\
n \rightarrow n^{\prime}
\end{gathered}
$$

abbreviates

$T_{1}$ is given in

$$
\frac{1 \rightarrow 1^{\prime}}{2 \rightarrow 2^{\prime}}, \ldots, \frac{1 \rightarrow 1^{\prime}}{n \rightarrow n^{\prime}}
$$


Definition 3.2 (transition system $T_{1}$ ). Let $s, s^{\prime}, \tilde{s} \in \mathscr{L}_{1}, a \in A, D \in \mathscr{D}_{e c t}$.

$$
\begin{gathered}
a \stackrel{a}{\rightarrow}_{D} E \\
\frac{s \stackrel{a}{\rightarrow}_{D} s^{\prime} \mid E}{s ; \tilde{s} \vec{D}_{D} s^{\prime} ; \tilde{s} \mid \tilde{s}} \\
\frac{s \stackrel{a}{\rightarrow}_{D} s^{\prime} \mid E}{s \cup \tilde{s} \stackrel{a}{D}_{D} s^{\prime} \mid E} \\
\tilde{s} \cup s \stackrel{a}{\rightarrow}_{D} s^{\prime} \mid E \\
\frac{g \stackrel{a}{\rightarrow}_{D} s^{\prime} \mid E}{x \stackrel{a}{\rightarrow}_{D} s^{\prime} \mid E}, \text { with } x \in g \text { in } D \\
\frac{s \stackrel{a}{\rightarrow}{ }_{D} s^{\prime} \mid E}{s \| \tilde{s} \stackrel{a}{\rightarrow} s^{\prime}|\tilde{s}| \tilde{s}} \\
\tilde{s}|| s \stackrel{a}{\rightarrow}_{D} \tilde{s}|| s^{\prime} \mid \tilde{s}
\end{gathered}
$$

We next define how to collect the successive transitions $s \stackrel{a}{\rightarrow}{ }_{D} s^{\prime}, s^{\prime} \stackrel{b}{\rightarrow} s^{\prime \prime}, \ldots$, starting from some $t \equiv\langle D \mid s\rangle$, into its operational meaning $\mathcal{O} \llbracket t \rrbracket$. We use $Q$ as introduced in section 2.3 .

\section{DEFINITION 3.3.}

a. The mapping $\mathcal{O}: \mathscr{P P}_{\text {rog }} \rightarrow Q$ is given by $\mathcal{O} \llbracket\langle D \mid s\rangle \rrbracket=\mathcal{O}_{D} \llbracket s \rrbracket$.

b. The mapping $\mathcal{O}_{D}: \mathscr{L}_{1}^{\prime} \rightarrow Q$ is given by: $\mathcal{O}_{D} \llbracket E \rrbracket=\{\varepsilon\}$, and for $s \neq E$,

$$
\mathcal{O}_{D} \llbracket s \rrbracket=\cup\left\{a \cdot \mathcal{O}_{D} \llbracket s^{\prime} \rrbracket \mid s \stackrel{a}{\rightarrow}_{D} s^{\prime}\right\},
$$

where the transitions are with respect to $T_{1}$. Also, $a \cdot \ldots$ denotes the usual prefixing operation: $a \cdot\{\varepsilon\}=\{a\}, a \cdot X=\{a \cdot u \mid u \in X\}$, where $a \cdot u$ is assumed to be known.

It may not be obvious that the function $\mathcal{O}_{D}$ is well-defined. This is in fact a consequence of the following

LeMma 3.4. Let the operator $\Phi:\left(\mathscr{L}_{1}^{\prime} \rightarrow Q\right) \rightarrow\left(\mathscr{L}_{1}^{\prime} \rightarrow Q\right)$ be defined as follows:

For any $\mathscr{F}_{D}: \mathscr{L}_{1}^{\prime} \rightarrow Q$ we put $\Phi\left(\mathscr{F}_{D}\right)(E)=\{\varepsilon\}$, and, for $s \neq E$,

$$
\Phi\left(\mathscr{F}_{D}\right)(s)=\cup\left\{a \cdot \mathscr{F}_{D} \llbracket s^{\prime} \rrbracket \mid s \stackrel{a}{\rightarrow}_{D} s^{\prime}\right\} .
$$

Then $\Phi$ is a contracting mapping with $\mathcal{O}_{D}$ as its fixed point.

Proof. Clear from the definitions and Banach's theorem. 
EXAMPLE. $\mathcal{O}[\langle x \Leftarrow a ; x \cup b \mid x\rangle]=\left\{a^{\omega}\right\} \cup a^{*} \cdot b$.

REMARK. As explained in [10], if we were to drop the guardedness restriction for the $g_{i}$ in $D$, the operational meaning of $\langle D \mid s\rangle$ (based on the definitions in $[10]$ ) is not necessarily a closed set, and definition 3.3 would not, in general, yield the desired result. (Definition 3.3 always gives closed sets as results.)

The next step is the development of the denotional model. We now also define various semantic operators: $Q \times Q \rightarrow Q$, viz. the operators of union $\left({ }^{\prime} \cup^{\prime}\right)$, composition ('o') and merge (' $\left.\mid l^{\prime}\right)$.

Definition 3.5. For each $X \in Q$ we write $X_{a}=\left\{u \in A^{\infty} \mid a \cdot u \in X\right\}$.

a. $X \cup Y$ equals the set-theoretic union of $X$ and $Y$.

b. Let $o p$ stand for $\circ$ or II. Let $\phi$ be any ndi mapping: $Q \times Q \rightarrow Q$. Let $\Phi_{o p}:(Q \times Q \rightarrow Q) \rightarrow(Q \times Q \rightarrow Q)$ be defined as follows :

$$
\begin{aligned}
& \Phi_{\circ}(\phi)(X)(Y)= \begin{cases}\cup_{a \in A}\left\{a \cdot \phi\left(X_{a}\right)(Y) \mid X_{a} \neq \phi\right\} \cup Y, & \text { if } \varepsilon \in X_{a} \\
\cup_{a \in A}\left\{a \cdot \phi\left(X_{a}\right)(Y) \mid X_{a} \neq \varnothing\right\}, & \text { otherwise }\end{cases} \\
& \Phi_{\|}(\phi)(X)(Y)=\Phi_{\circ}(\phi)(X)(Y) \cup \Phi_{\circ}(\phi)(Y)(X)
\end{aligned}
$$

c. We now put $\circ=$ fixed point $\left(\Phi_{\circ}\right), \|=$ fixed point $\left(\Phi_{\|}\right)$.

We have

LEMMA 3.6. The operators $\cup, \circ, \|$ are well-defined and ndi (and, hence, continuous).

Proof. Clear for $U$. For the operators, another appeal to Banach's theorem together with some calculations in the style of Appendix B of [11] suffices.

The denotational semantic definitions employ the usual notion of environment. Let $(\gamma \in) \Gamma=\mathscr{P} v a r \rightarrow Q$ be the set of environments, i.e. of mappings from procedure variables to their meanings. We define

Definition 3.7 (denotational semantics for $\mathscr{L}_{1}, \mathscr{P}_{\text {ro }} g_{1}$ ). Below we often suppress parentheses around arguments of functions. The mappings $\mathscr{M}: \mathscr{P}_{\text {rog }} \rightarrow Q$ and $\mathscr{D}: \mathscr{L}_{1} \rightarrow(\Gamma \rightarrow Q)$ are given as follows :

a. $\mathscr{M} \llbracket\langle D \mid s\rangle \rrbracket=\mathscr{D} \llbracket s \rrbracket \gamma_{D}$, with $\gamma_{D}$ as in clause b.

b. $\gamma_{D}=\gamma\left\{X_{i} / x_{i}\right\}_{i=1}^{n}$, where $\gamma$ is arbitrary, and, for $D \equiv\left\langle x_{i} \Leftarrow g_{i}\right\rangle_{i}$, we put

$$
\left\langle X_{1}, \ldots, X_{n}\right\rangle=\text { fixed point }\left\langle\Phi_{1}, \ldots, \Phi_{n}\right\rangle
$$

, where $\Phi_{j}: Q^{n} \rightarrow Q$ is given by $\Phi_{j}\left(Y_{1}\right) \ldots\left(Y_{n}\right)=\mathscr{D} \llbracket g_{j} \rrbracket \gamma\left\{Y_{i} / x_{i}\right\}_{i}$. 
c. $\mathscr{D} \llbracket a \rrbracket \gamma=\{a\}, \mathscr{D} \llbracket x \rrbracket \gamma=\gamma(x), \mathscr{D} \llbracket s_{1} \underline{o p} s_{2} \rrbracket \gamma=\mathscr{D} \llbracket s_{1} \rrbracket \gamma o p \mathscr{D} \llbracket s_{2} \rrbracket \gamma$, for $\underline{o p}=; \cup, \|$ and $o p=\circ, \cup, \|$, respectively.

EXAMPLES. $\mathscr{D} \llbracket a ;(b \cup c) \rrbracket \gamma=\mathscr{D} \llbracket(a ; b) \cup(a ; c) \rrbracket \gamma=\{a b, a c\}$.

$\mathscr{M} \llbracket\langle x \Leftarrow a ;(b \| x) \mid x\rangle \rrbracket=\lim _{i} X_{i}$, where $X_{i+1}=a \cdot\left(b \| X_{i}\right)$, and $X_{0} \in Q$ is arbitrary.

The well-definedness of $\mathscr{D}$ is a consequence of the guardedness requirement which ensures the contractivity of the $\Phi_{j}$, whence the fixed points in clause b exist :

LemMA 3.8. Assume the notation as in definition 3.7. For simplicity, we take $n=1$.

a. For each $s \in \mathscr{L}_{1}$,

$$
\mathbf{d}\left(\mathscr{D} \llbracket s \rrbracket \gamma\left\{Y_{1} / x\right\}, \mathscr{D} \llbracket s \rrbracket \gamma\left\{Y_{2} / x\right\}\right) \leqq \mathbf{d}\left(Y_{1}, Y_{2}\right)
$$

b. For each $g \in \mathscr{L}_{1}^{g}$,

$$
\mathbf{d}\left(\mathscr{D} \llbracket g \rrbracket \gamma\left\{Y_{1} / x\right\}, \mathscr{D} \llbracket g \rrbracket \gamma\left\{Y_{2} / x\right\}\right) \leqq \frac{1}{2} \cdot \mathbf{d}\left(Y_{1}, Y_{2}\right) .
$$

Proof. We use induction on the complexity of $s$ and $g$. We give details of a few subcases for $g$ :

- $g=g_{1} ; s$ :

We use the abbreviation $Y_{i}^{\prime}=\mathscr{D} \llbracket g_{1} \rrbracket \gamma\left\{Y_{i} / x\right\}$ for $i=1,2$. Now

$$
\begin{aligned}
& \mathbf{d}\left(\mathscr{D} \llbracket g_{1} ; s \rrbracket \gamma\left\{Y_{1} / x\right\}, \mathscr{D} \llbracket g_{1} ; s \rrbracket \gamma\left\{Y_{2} / x\right\}\right)= \\
& \mathbf{d}\left(Y_{1}^{\prime} \circ \mathscr{D} \llbracket s \rrbracket \gamma\left\{Y_{1} / x\right\}, Y_{2}^{\prime} \circ \mathscr{D} \llbracket s \rrbracket \gamma\left\{Y_{2} / x\right\}\right) .
\end{aligned}
$$

If $Y_{1}^{\prime} \neq Y_{2}^{\prime}$, we use the induction hypothesis and the fact that, in general, for $Z_{1} \neq U_{1}$,

$$
\mathbf{d}\left(Z_{1} \circ Z_{2}, U_{1} \circ U_{2}\right) \leqq \mathrm{d}\left(Z_{1}, U_{1}\right) .
$$

If $Y_{1}^{\prime}=Y_{2}^{\prime}$, we use part a, and the fact that, for $\varepsilon \notin Z$,

$$
\mathbf{d}\left(Z \circ Z_{2}, Z \circ U_{2}\right) \leqq \frac{1}{2} \cdot \mathbf{d}\left(Z_{2}, U_{2}\right) .
$$

- $g=g_{1} \| g_{2}$ :

We use the abbreviations $Y_{i j}=\mathscr{D} \llbracket g_{i} \rrbracket \gamma\left\{Y_{j} / x\right\}$ for $i, j \in\{1,2\}$. Now

$$
\begin{aligned}
& \mathbf{d}\left(\mathscr{D} \llbracket g_{1}\left\|g_{2} \rrbracket \gamma\left\{Y_{1} / x\right\}, \mathscr{D} \llbracket g_{1}\right\| g_{2} \rrbracket \gamma\left\{Y_{2} / x\right\}\right)= \\
& \mathbf{d}\left(Y_{11}\left\|Y_{21}, Y_{12}\right\| Y_{22}\right) \leqq \\
& \max \left(\mathbf{d}\left(Y_{11}, Y_{12}\right), \mathbf{d}\left(Y_{21}, Y_{22}\right)\right) \leqq \\
& \frac{1}{2} \cdot \mathbf{d}\left(Y_{1}, Y_{2}\right),
\end{aligned}
$$

where we have used that, in general,

$$
\mathrm{d}\left(Z_{1}\left\|Z_{2}, U_{1}\right\| U_{2}\right) \leqq \max \left(\mathrm{d}\left(Z_{1}, U_{1}\right), \mathrm{d}\left(Z_{2}, U_{2}\right)\right),
$$

and the induction hypothesis (twice). 
The above definitions of the operational and denotational semantics have been tuned such that the proof of $\mathscr{O}=\mathscr{M}$ is now no longer a major undertaking (as it was in [10]). We follow the approach as in [18] (cf. [16], [5] for a similar approach in an order-theoretic framework) with the additional simplifications due to our replacing $\mu$-constructs by simultaneous recursion. We prove

THEOREM 3.9. For all $t \in \mathscr{P}_{\text {rog }}, \mathcal{O} \llbracket t \rrbracket=\mathscr{M} \llbracket t \rrbracket$.

Proof. Let us put $\mathscr{D}_{D} \llbracket s \rrbracket={ }^{d f} \cdot \mathscr{D} \llbracket s \rrbracket \gamma_{D}$. By the definition of $\mathcal{O}$ and lemma 3.4 , it is sufficient to show that, for $s \in \mathscr{L}_{1},\left(^{*}\right): \mathscr{D}_{D} \llbracket s \rrbracket=\Phi\left(\mathscr{D}_{D}\right)(s)$. The proof proceeds in two stages; first for $g \in \mathscr{L}_{1}^{g}$ and next for any $s \in \mathscr{L}_{1}$.

Stage 1. Take $g \in \mathscr{L}_{1}^{q}$. We prove $\left({ }^{*}\right)$ by induction on the complexity of $g$. We only treat the case that $g=g_{1} \| g_{2}$, the other cases being simpler. We have:

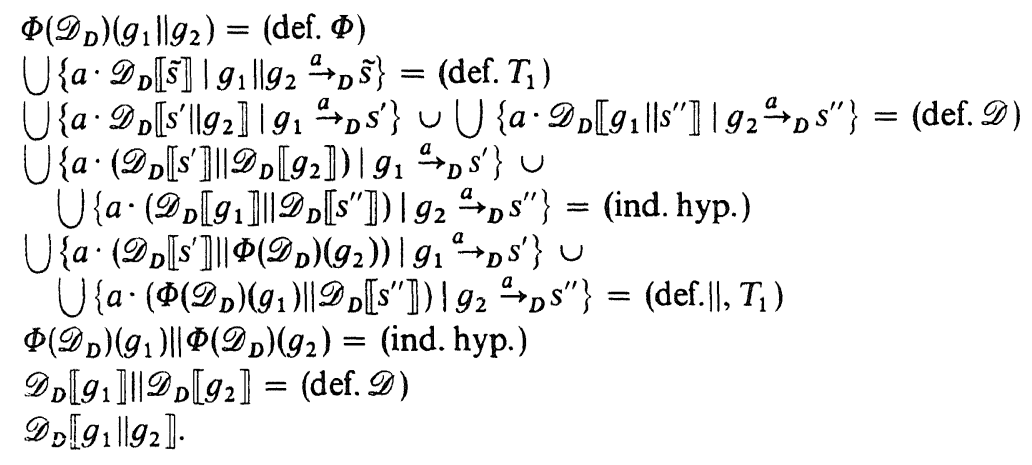

Stage 2. Take $s \in \mathscr{L}_{1}$. We prove $\left({ }^{*}\right)$ by induction on the complexity of $s$. All cases are as in stage 1 , but for the case $s \equiv x$, with $x \equiv x_{i} \in \mathscr{X}$. We have

$\Phi\left(\mathscr{D}_{D}\right)\left(x_{i}\right)=\bigcup\left\{a \cdot \mathscr{D}_{D} \llbracket \tilde{s} \rrbracket \mid x_{i} \stackrel{a}{\rightarrow}_{D} \tilde{s}\right\}=\bigcup\left\{a \cdot \mathscr{D}_{D} \llbracket \tilde{s} \rrbracket \mid g_{i} \stackrel{a}{\rightarrow}{ }_{D} \tilde{s}\right\}$ (with $x_{i} \Leftarrow g_{i}$ in $\left.\mathscr{D}\right)$ $=$ (by stage 1 , the desired result holds for $\left.g_{i} \in \mathscr{L}_{1}^{g}\right) \mathscr{D}_{D} \llbracket g_{i} \rrbracket=$ (by the fixed point property) $\mathscr{D}_{D} \llbracket x_{i} \rrbracket$.

REMARK. Now that we have developed the appropriate metric semantics machinery, we can elaborate on the reasons why the concepts of fairness and hiding are not (directly) amenable to a metric approach. For fairness, note that $a^{\omega} \|_{\text {fair }} b^{\omega}=\left(a^{*} b b^{*} a\right)^{\omega}$, which is not a closed set. (It does not include, e.g., the limit $a^{\omega}$.) For hiding, note that, e.g., $X=\left\{a^{n} b^{n} \mid n \geqq 1\right\} \cup\left\{a^{\omega}\right\}$ is closed, whereas $X$ hid $a$ is not. ( $X$ hid $a \supseteq b^{*}$, but $b^{\omega} \notin X$ hid $a$.)

\section{Synchronization and global nondeterminacy.}

We discuss an extension of $\mathscr{L}_{1}$ with two new features. Firstly, we add a form of synchronization in the tradition of CCS [22] or CSP [17]. Secondly, we replace the nondeterministic choice $\left(s_{1} \cup s_{2}\right)$ of section 3 by a new form of 
nondeterminism, written as $s_{1}+s_{2}$. The latter is called global (sometimes also external) nondeterminism. In the presence of synchronization, the former variety is then called local. For an extensive discussion of these two notions we refer to [10] and the papers cited there. The interesting point with the notion of global nondeterminacy is that it needs some form of non-LT denotational semantics to make sufficient distinctions. For example, assuming that $a, b$ are normal actions and $c$ is a communication action (which requires a corresponding $\bar{c}$ in a parallel component to establish synchronization), we want to assign different denotational meanings to $s_{1} \equiv a ;(b+c)$ and $s_{2} \equiv(a ; b)+(a ; c)$. A simple LT model would not capture the operational intuition (which treats $s_{1}, s_{2}$ differently, details follow), since it would deliver the outcome $\{a b, a c\}$ in both cases (cf. the example following definition 3.7).

We shall present below a branching time (BT) denotational model for $\mathscr{L}_{2}$ which indeed provides the desired refinement to distinguish between $\mathscr{D} \llbracket s_{1} \rrbracket$ and $\mathscr{D} \llbracket s_{2} \llbracket$.

The syntax and operational semantics for $\mathscr{L}_{2}$ exhibit only minor differences with those for $\mathscr{L}_{1}$. Firstly, we assume a subset $(c \in) C \leqq A$ of communications, and assume moreover a mapping ${ }^{-}: C \rightarrow C$, such that (writing $\bar{c}$ for ${ }^{-}(c)$ ) we have $\bar{c}=c$. Finally, we postulate a special element $\tau \in A \backslash C$ which will be used as outcome for a successful synchronization between an action $c$ and its counterpart $\bar{c}$. Fot this we refer to the rule(s) Synch in definition 4.2.

Definition 4.1 (Syntax). Let $A$ be as just described, and $\mathscr{X}$ as in section 3.

a $\quad\left(s \in \mathscr{L}_{2}\right) . \quad s::=a|x| s_{1} ; s_{2}\left|s_{1}+s_{2}\right| s_{1}|| s_{2}$, with $x \in \mathscr{X}$.

b $\quad\left(g \in \mathscr{L} \mathscr{L}_{2}\right) . g::=a|g ; s| g_{1}+g_{2} \mid g_{1} \| g_{2}$.

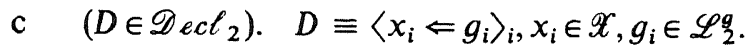

$\mathrm{d} \quad\left(t \in \mathscr{P}_{\text {rog }_{2}}\right) . \quad t \equiv\langle D \mid s\rangle, D \in \mathscr{D}_{\text {ect }}, s \in \mathscr{L}_{2}$.

e $\quad \mathscr{L}_{2}^{\prime}=\mathscr{L}_{2} \cup\{E\}$.

The transition system $T_{2}$ is given in

Defintion 4.2. The transition system $T_{2}$ contains Elem, SeqComp, Rec and ParComp from $T_{1}$. Moreover, it contains the rules $\left(s, s^{\prime}, \tilde{s}, s_{1}, s_{2}, s^{\prime \prime} \in \mathscr{L}_{2}, a \in A\right.$, $c \in C, D \in \mathscr{D e c l}_{2}$ )

$$
\begin{gathered}
\frac{s \stackrel{a}{\rightarrow}_{D} s^{\prime} \mid E}{s+\tilde{s} \stackrel{a_{D}}{s^{\prime} \mid E}} \\
\tilde{s}+s \stackrel{a}{\rightarrow} D_{D} s^{\prime} \mid E \\
\frac{s_{1} \stackrel{c}{\rightarrow}_{D} s^{\prime}, s_{2} \stackrel{\bar{c}}{\rightarrow}{ }_{D}^{\prime \prime}}{s_{1}\left\|s_{2} I_{D} s^{\prime}\right\| s^{\prime \prime}}
\end{gathered}
$$




$$
\begin{aligned}
& \frac{s_{1} \stackrel{c}{\rightarrow}{ }_{D} s^{\prime}, s_{2} \stackrel{\bar{c}}{\rightarrow} D}{s_{1} \| s_{2} \dot{L}_{D} s^{\prime}} \text {, and a symmetric rule } \quad\left(\text { Synch }_{2}\right) \\
& \frac{s_{1} \stackrel{c}{\rightarrow} D E, s_{2} \stackrel{\bar{c}}{\rightarrow} D}{s_{1} \| s_{2} \stackrel{\check{L}}{\rightarrow}_{D} E}
\end{aligned}
$$

\section{REMARKS.}

1. By Elem, we now also have that $c \stackrel{c}{\rightarrow}_{D} E$.

2. (In contrast to [10].) The rules in $T_{1}$ and $T_{2}$ for ' $\cup^{\prime}$ and ' + ', respectively, have the same form. The difference will become manifest when we define $\mathcal{O}_{D}$ for $s$ in $\mathscr{L}_{2}$.

In order to define $\mathcal{O}$ for $\mathscr{P}_{\text {rog }}$, we provide a slight variation on the set $Q$ used in section 3 . We introduce a new symbol $\delta \notin A$, modelling failure, and we put $A_{\delta}^{\infty}=A^{*} \cup A^{\omega} \cup A^{*} \cdot \delta$. Thus, $A_{\delta}^{\infty}$ extends $A^{\infty}$ by adding all finite sequences over $A$ to which $\delta$ is appended. Furthermore, we put $R=\mathscr{P}_{n c}\left(A_{\delta}^{\infty}\right)$. We shall again use $X, Y$ to range over $R$, and use the notation $X_{a}$ as before. (Note, however, that elements in $X_{a}$ now may end with $\delta$.) We give

DefinIrion 4.3 (operational semantics for $\mathscr{P}_{\text {rog }}, \mathscr{L}_{2}$ ).

a. $\quad \mathcal{O}: \mathscr{P P r o g}_{2} \rightarrow R$ is given by $\mathcal{O} \llbracket\langle D \mid s\rangle \rrbracket=\mathcal{O}_{D} \llbracket s \rrbracket$.

b. $\quad \mathcal{O}_{D}: \mathscr{L}_{2}^{\prime} \rightarrow R$ is given by: $\mathcal{O}_{D} \llbracket E \rrbracket=\{\varepsilon\}$, and for $s \neq E$,

$$
\mathcal{O}_{D} \llbracket s \rrbracket=\left\{\begin{array}{l}
\{\delta\}, \text { if }\left\{a \mid s \stackrel{a}{\rightarrow} s^{\prime}, a \notin C\right\}=\varnothing \\
\bigcup\left\{a \cdot \mathcal{O}_{D} \llbracket s^{s^{\prime}} \rrbracket \mid s \stackrel{a}{\rightarrow}{ }_{D} s^{\prime}, a \notin C\right\}, \text { otherwise }
\end{array}\right.
$$

where the transitions are with respect to $T_{2}$.

As in section $3, \mathcal{O}_{D}$ may be shown to be well-defined by a contractivity argument.

EXAMPLE. $\mathcal{O}_{D} \llbracket a ;(b+c) \rrbracket=\{a b\}, \mathcal{O}_{D} \llbracket(a ; b)+(a ; c) \rrbracket=\{a b, a \delta\}$.

The denotational model for $\mathscr{L}_{2}$ assumes a domain $(p \in) P$ of branching time processes (cf. section 2.4) satisfying the isometry

$$
P \cong\left\{p_{0}\right\} \cup \mathscr{P}_{\text {closed }}\left(A \times i d_{1 / 2}(P)\right)
$$


Here we assume the discrete metric on $A$. Typical processes are

- the "nil process" $p_{0}$ and the empty process $\phi$ (the empty set), corresponding to the LT objects $\{\varepsilon\}$ and $\{\delta\}$, respectively,

- $\left\{\left\langle a,\left\{\left\langle b, p_{0}\right\rangle\right\}\right\rangle,\left\langle a,\left\{\left\langle c, p_{0}\right\rangle\right\}\right\rangle\right\}$, which is different from $\left\{\left\langle a,\left\{\left\langle b, p_{0}\right\rangle,\left\langle c, p_{0}\right\rangle\right\}\right\rangle\right\}$,

- the infinite process $p=\lim _{n} p_{n}$, where $p_{n+1}=\left\{\left\langle a, p_{n}\right\rangle,\left\langle b, p_{n}\right\rangle\right\}$.

We recall from section 2.4 that $P$ is a complete ultrametric space, and that elements of $P$ are either finite or satisfy $p=\lim _{n} p_{n}$, for $p_{n}$ finite. We draw attention to the difference between $\{\delta\} \in R$ and $\phi \in P$. There is no problem in incorporating $\varnothing$ into $P$. In particular, we have that $\mathrm{d}\left(\left\{\left\langle a, p_{1}\right\rangle\right\},\left\{\left\langle a, p_{2}\right\rangle\right\}\right)$ $=\frac{1}{2} \cdot \mathbf{d}\left(p_{1}, p_{2}\right)$ holds, even for $p_{1}$ or $p_{2}$ equal to $\phi$. (This follows from the use of $i d_{1 / 2}(P)$ on the right-hand side of (4.1).) On the other hand, since $a \cdot \phi=\phi$, including $\phi$ into $R$ would invalidate the contractivity property $\mathrm{d}\left(a \cdot X_{1}, a \cdot X_{2}\right)$ $=\frac{1}{2} \cdot \mathrm{d}\left(X_{1}, X_{2}\right)$.

We next define the semantic operators $o p: P \times P \rightarrow P$, for $o p \in\{\cup, \circ, \|\}$, as natural variations on those of definition 3.5 .

\section{DefinITION 4.4.}

a. $\quad p \cup q=p$, if $q=p_{0}$,

$p \cup q=q$, if $p=p_{0}$.

Otherwise, $p \cup q$ equals the set-theoretic union of the sets $p$ and $q$.

b. Let $o p$ stand for $\circ$ or $\|$. Let $\phi$ be any ndi mapping: $P \times P \rightarrow P$. Let $\Phi_{o p}:(P \times P \rightarrow P) \rightarrow(P \times P \rightarrow P)$ be defined as follows:

$$
\begin{aligned}
& \Phi_{\mathrm{o}}(\phi)(p)(q)=\left\{\begin{array}{l}
q, \text { if } p=p_{0} \\
\left\{\left\langle a, \phi\left(p^{\prime}\right)(q)\right\rangle \mid\left\langle a, p^{\prime}\right\rangle \in p\right\}, \text { otherwise }
\end{array}\right. \\
& \Phi_{\|}(\phi)(p)(q)=\Phi_{\mathrm{o}}(\phi)(p)(q) \cup \Phi_{\mathrm{o}}(\phi)(q)(p) \cup \hat{\phi}_{\mid}(p)(q)
\end{aligned}
$$

where $\hat{\phi}_{1}^{\prime}: P \times P \rightarrow P$ is given by

$$
\hat{\phi}(p)(q)=\left\{\left\langle\tau, \phi\left(p^{\prime}\right)\left(q^{\prime}\right)\right\rangle \mid\left\langle c, p^{\prime}\right\rangle \in p,\left\langle\bar{c}, q^{\prime}\right\rangle \in q\right\}
$$

c. We now put $\circ=$ fixed point $\left(\Phi_{0}\right), \|=$ fixed point $\left(\Phi_{\|}\right)$.

We have again that the operators $\cup, \circ, \|$ are well-defined and ndi (and, hence, continuous).

The denotational definitions are now easy variations on the ones in section 3 . Let $(\gamma \in) \Gamma_{2}=\mathscr{X} \rightarrow P$. Now $\mathscr{M}: \mathscr{P}_{\text {rog }_{2}} \rightarrow P$ and $\mathscr{D}: \mathscr{L}_{2} \rightarrow\left(\Gamma_{2} \rightarrow P\right)$ are defined in 
Definition 4.5 (denotational semantics for $\mathscr{L}_{2}, \mathscr{P}_{\text {ro }} \mathrm{g}_{2}$ ).

a. $\mathscr{M} \llbracket\langle D \mid s\rangle \rrbracket=\mathscr{D} \llbracket s \rrbracket \gamma_{D}$.

b. $\gamma_{D}=\gamma\left\{p_{i} / x_{i}\right\}_{i=1}^{n}$, where, for $D \equiv\left\langle x_{i} \Leftarrow g_{i}\right\rangle_{i}$, we put

$$
\left\langle p_{1}, \ldots, p_{n}\right\rangle=\text { fixed point }\left\langle\Phi_{1}, \ldots, \Phi_{n}\right\rangle
$$

with $\Phi_{j}: P^{n} \rightarrow P$ is given by, for $j=1, \ldots, n, \Phi_{j}\left(q_{1}\right) \ldots\left(q_{n}\right)=\mathscr{D} \llbracket \llbracket g_{j} \rrbracket \gamma\left\{q_{i} / x_{i}\right\}_{i}$.

c. $\mathscr{D} \llbracket a \rrbracket \gamma=\left\{\left\langle a, p_{0}\right\rangle\right\}, \mathscr{D} \llbracket x \rrbracket \gamma=\gamma(x)$,

$\mathscr{D} \llbracket s_{1} \underline{o p} s_{2} \rrbracket \gamma=\mathscr{D} \llbracket s_{1} \rrbracket \gamma o p \mathscr{D} \llbracket s_{2} \rrbracket \gamma$, for $\underline{o p}=; \cup, \|$ and $o p=\circ, \cup, \|$, respectively.

EXAMPLES. $\mathscr{D} \llbracket a ;(b+c) \rrbracket \gamma=\left\{\left\langle a,\left\{\left\langle b, p_{0}\right\rangle,\left\langle c, p_{0}\right\rangle\right\}\right\rangle\right\}$, $\mathscr{D} \llbracket(a ; b)+(a ; c) \rrbracket \gamma=\left\{\left\langle a,\left\{\left\langle b, p_{0}\right\rangle\right\}\right\rangle,\left\langle a,\left\{\left\langle c, p_{0}\right\rangle\right\}\right\rangle\right\}$,

$\mathscr{M} \llbracket\langle x \Leftarrow a ;(b \| x) \mid x\rangle \rrbracket=\lim _{i} p_{i}$, where $p_{i+1}=\left\{\left\langle a,\left\{\left\langle b, p_{0}\right\rangle\right\} \| p_{i}\right\rangle\right\}$.

We see that $\mathscr{D} \llbracket s \rrbracket \gamma$ contains traces of unsuccessful communications which are not present in $\mathcal{O}_{D} \llbracket s \rrbracket$. For example, $\mathscr{D} \llbracket c \rrbracket \gamma=\left\{\left\langle c, p_{0}\right\rangle\right\}, \mathcal{O}_{D} \llbracket c \rrbracket=\delta$. Moreover, the elements delivered by $\mathscr{D}[s] \gamma$ are branching time objects (in $P$ ) and the elements delivered by $\mathcal{O}_{D} \llbracket s \rrbracket$ are linear time objects (in $R$ ). We therefore define an abstraction operator abs: $P \rightarrow R$ which links the two meanings: given an argument $p, a b s$ deletes $\langle c, \ldots\rangle$ branches from $p$, and collapses the branching time structure into the set of all "paths" in the process $p$.

Definition 4.6 (abstraction). We define $a b s$ as fixed point of the contracting mapping $\Psi_{a b s}:(P \rightarrow R) \rightarrow(P \rightarrow R)$ given as follows: Let $\psi \in P \rightarrow R$. Writing $\tilde{\psi}_{\text {abs }}$ as shorthand for $\Psi_{\text {abs }}(\psi)$, we put

and, for $p \neq p_{0}$,

$$
\tilde{\psi}_{a b s}\left(p_{0}\right)=\{\varepsilon\}
$$

$$
\tilde{\psi}_{a b s}(p)=\left\{\begin{array}{l}
\{\delta\}, \text { if }\left\{a \mid\left\langle a, p^{\prime}\right\rangle \in p, a \notin C\right\}=\varnothing \\
\bigcup\left\{a \cdot \psi\left(p^{\prime}\right) \mid\left\langle a, p^{\prime}\right\rangle \in p, a \notin C\right\}, \text { otherwise. }
\end{array}\right.
$$

REMARK. The well-definedness of $a b s$ relies on the finiteness of $A$, cf. [6].

It can now be shown that

THEOREM 4.7. For each $t \in \mathscr{P P}_{\mathrm{rog}_{2}}, \mathcal{O} \llbracket t \rrbracket=(a b s \circ \mathscr{M}) \llbracket t \rrbracket$.

We omit the proof which is an extension of that of theorem 3.9. Details are given in [18]. 


\section{Process creation.}

We now turn to the study of a simple uniform language with process ${ }^{\dagger}$ creation as central feature. We couch the notion of process creation in the framework of the language $\mathscr{L}_{3}$ (with induced $\mathscr{P} \mathfrak{r g}_{3}$ ). This language is like $\mathscr{L}_{1}$ (or $\left.\mathscr{P}_{\mathrm{r} \mathrm{g}_{1}}\right)$, but with the construct of merge replaced by the construct new $(s)$ : execution of new(s) creates a new process with body $s$, to be executed in parallel with the already existing processes. (A more precise definition follows in a moment.)

We first encountered the notion of process creation during our study of the semantics of POOL, a parallel object-oriented language. In $[2,3]$ we have designed operational and denotational semantics for POOL, and in [1] we massaged these definitions such that the equivalence of the two semantics for process creation could be shown. What follows below is a new presentation, which could be simplified considerably thanks to another application of a contractivity argument.

We assume $A$ and $\mathscr{X}$ as in section 3. (For simplicity, this section has no $(c \in) C \cong A$, and ' $\cup$ ' again replaces ' + '.)

\section{Definimion 5.1 (Syntax).}

a $\quad\left(s \in \mathscr{L}_{3}\right) . \quad s::=a|x| s_{1} ; s_{2}\left|s_{1} \cup s_{2}\right|$ new $(s)$, with $x \in \mathscr{X}$

b $\quad\left(s \in \mathscr{L}_{3}\right) . \quad g::=h\left|g_{1} ; g_{2}\right| g_{1} \cup g_{2} \mid$ new $(g)$

$(h \in H) . \quad h::=a|h ; s| h_{1} \cup h_{2}$,

c $\left(D \in \mathscr{D}_{e c \mathcal{\ell}_{3}}\right) . \quad D \equiv\left\langle x_{i} \Leftarrow g_{i}\right\rangle_{i}, x_{i} \in \mathscr{X}, g_{i} \in \mathscr{L}_{3}^{g}, i=1, \ldots, n$.

$\mathrm{d} \quad\left(t \in \mathscr{P}_{\mathrm{Pog}}\right) . \quad t \equiv\langle D \mid s\rangle, D \in \mathscr{D}_{\text {ect }}, s \in \mathscr{L}_{3}$.

Remark. The complications in the definition of $(g \in) \mathscr{L}^{\mathbb{b}}$ are caused by the following phenomenon: We want to make sure that occurrences of $x$ in $g$ are guarded by some statement which starts with an elementary action $a$. Without the precaution as taken in clause b (i.e., adopting a syntax for $\mathscr{L}_{3}^{g}$, analogous to $\mathscr{L}_{1}^{g}$, of the form $g::=a|g ; s| g_{1} \cup g_{2} \mid$ new $\left.(g)\right)$, a statement new $(a) ; x$ would qualify as guarded. As we shall see later, the intended meaning of new $(a) ; x$ is the same as that of the unguarded $\left(\mathscr{L}_{1}^{-}\right)$statement $a \| x$, allowing execution of $x$ before $a$. This would violate the desired contractivity of the function(s) associated with the declarations; hence, the need for the more involved definition.

Before providing the formal semantic definitions, we first present an informal explanation of process creation. The execution of $s$ is described in terms of a

† The programming notion of "process" as studied in section 5 has nothing to do with the mathematical notion of "process" appearing in section 4. 
dynamically growing number of processes which execute statements in parallel in the following manner (all steps are with respect to some given $D$ ):

1. Set an auxiliary variable $i$ to 1 and set $s_{1}$ to $s$, the statement to be executed. A process, numbered 1 , is created to execute $s_{1}$.

2. Processes 1 to $i$ execute in parallel. Process $j$ executes $s_{j}(1 \leqq j \leqq i)$ in the usual way in case $s_{j}$ does not begin with some new $\left(s^{\prime}\right)$ statement.

3. If some process $j(1 \leqq j \leqq i)$ has to execute a statement of the form new $\left(s^{\prime}\right)$, then the variable $i$ is set to $i+1, s_{i}$ is set to $s^{\prime}$, and a new process with number $i$ is created to execute $s_{i}$. Process $j$ will continue to execute the part after the new $\left(s^{\prime}\right)$ statement. Go to step 2.

4. Execution terminates if all processes have terminated their execution.

We proceed with the formal semantic definitions. We use a somewhat extended transition formalism which involves constructs defined in

\section{Definition 5.2.}

a. The set $(r \in) \mathscr{S}_{e q}$ of sequents is defined by $r::=E \mid s ; r$, with $s \in \mathscr{L}_{3}$.

b. The set $(\varrho \in) \mathscr{P}$ ar of parallel constructs is defined by $\varrho::=r_{1}, \ldots, r_{n}, n \geqq 1$.

Transitions in $T_{3}$ are elements of $\mathscr{P} a r \times A \times \mathscr{D}_{e c} \ell_{3} \times \mathscr{P}_{a}$, written in the notation

$$
\varrho \stackrel{a}{\rightarrow} \varrho_{D} \text {. }
$$

We shall often encounter instances of transitions written as $\ldots, r, \ldots \stackrel{a}{\rightarrow} D, \ldots, r^{\prime}, \ldots$. Here $r\left(r^{\prime}\right)$ is a component of $\varrho\left(\varrho^{\prime}\right)$, and the notation implies that all terms at the dots (...) are unaffected by the transition. Mutatis mutandis, such notation also applies to transition rules.

Definition 5.3 (transition system $T_{3}$ ).

$$
\begin{aligned}
& \ldots, a ; r, \ldots \stackrel{a}{\rightarrow}_{D} \ldots, r, \ldots \\
& \frac{\ldots, s_{1} ;\left(s_{2} ; r\right), \ldots \stackrel{a}{\rightarrow} \varrho \varrho}{\ldots,\left(s_{1} ; s_{2}\right) ; r, \ldots \stackrel{a}{\rightarrow}_{D} \varrho} \\
& \frac{\ldots, s ; r, \ldots \stackrel{a}{\rightarrow}_{D} \varrho}{\ldots,(s \cup \tilde{s}) ; r, \ldots{\stackrel{a}{a_{D}}} \varrho} \\
& \ldots,(\tilde{s} \cup s) ; r, \ldots \stackrel{a}{\rightarrow} D \varrho \\
& \frac{\ldots, g ; r, \ldots \stackrel{a}{\rightarrow}_{D} \varrho}{\ldots, x ; r, \ldots \stackrel{a}{\rightarrow}_{D} \varrho} \text {, with } x \Leftarrow g \text { in } D
\end{aligned}
$$

(SeqComp)

(Choice) 


$$
\frac{\ldots, r, \ldots, s ; E \stackrel{a}{\rightarrow}_{D} \varrho}{\ldots, \text { new }(s) ; r, \ldots \stackrel{a}{\rightarrow}_{D} \varrho}
$$

$(N e w)$

Note that in the rule ( $N e w$ ), if the transition in the consequence has $n$ components on its lefthand side, then the transition in the premise has $n+1$ components on its left-hand side.

EXAMPLE. new $(a ; \operatorname{new}(b, c)) ; d ; E \stackrel{a}{\rightarrow}{ }_{D} d ; E$, new $(b ; c) ; E \stackrel{b}{\rightarrow} \stackrel{D}{D}^{\circ}$

$d ; E, E, c ; E \stackrel{d}{\rightarrow}_{D} E, E, c ; E \stackrel{c}{\rightarrow}_{D} E, E, E$.

The operational semantics associated with $T_{3}$ is described in

DEFINITION 5.4 .

a. $\quad \mathcal{O}: \mathscr{P}_{\mathrm{rog}_{3}} \rightarrow Q$ is given by $\mathcal{O} \llbracket\langle D \mid s\rangle \rrbracket=\mathcal{O}_{D} \llbracket[s ; E \rrbracket$.

b. $\mathcal{O}_{D}: \mathscr{P}_{\text {ar }} \rightarrow Q$ is given by :

$$
\mathcal{O}_{D} \llbracket \varrho \rrbracket=\left\{\begin{array}{l}
\{\varepsilon\}, \text { if } \varrho=E, E, \ldots, E \\
\cup\left\{a \cdot \mathcal{O}_{D} \llbracket \llbracket \varrho^{\prime} \rrbracket \mid \varrho \stackrel{a}{\rightarrow}_{D} \varrho^{\prime}\right\}, \text { otherwise }
\end{array}\right.
$$

where the transitions are with respect to $T_{3}$.

REMARK. Well-definedness of $\mathcal{O}_{D}$ follows as usual.

We continue with the denotational definitions. Let $Q$ and the operators $\cup, \|$ be as in section 3 ("o" plays no role here). Besides the usual environments, we also introduce the set of socalled continuations $\mathscr{C}_{0}$ nt which, in the present setting, coincides with $Q$. We have, altogether, the following domains and functions:

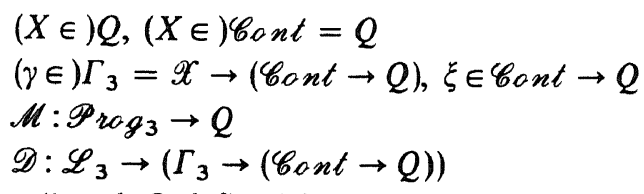

with $\mathscr{M}$ and $\mathscr{D}$ defined in

\section{DEFINITION 5.5.}

a. $\mathscr{M} \llbracket\langle D \mid s\rangle \rrbracket=\mathscr{D} \llbracket s \rrbracket \gamma_{D}\{\varepsilon\}$.

b. $\gamma_{D}=\gamma\left\{\xi_{i} / x_{i}\right\}_{i=1}^{n}$, where, for $D \equiv\left\langle x_{i} \Leftarrow g_{i}\right\rangle_{i}$, we put

$$
\left\langle\xi_{1}, \ldots, \xi_{n}\right\rangle=\text { fixed point }\left\langle\Phi_{1}, \ldots, \Phi_{n}\right\rangle
$$

with $\Phi_{j}:\left(\mathscr{C}_{0} n t \rightarrow Q\right)^{n} \rightarrow\left(\mathscr{C}_{0} n t \rightarrow Q\right)$ is given by $\Phi_{j}\left(\xi_{1}^{\prime}\right) \ldots\left(\xi_{n}^{\prime}\right)=\mathscr{D} \llbracket g_{j} \rrbracket \gamma\left\{\xi_{i}^{\prime} / x_{i}\right\}_{i}$, for $j=1, \ldots, n$. 
c. $\mathscr{D} \llbracket a \rrbracket \gamma X=a \cdot X, \mathscr{D} \llbracket x \rrbracket \gamma X=\gamma(x) X, \mathscr{D} \llbracket s_{1} ; s_{2} \rrbracket \gamma X=\mathscr{D} \llbracket s_{1} \rrbracket \gamma\left(\mathscr{D} \llbracket s_{2} \rrbracket \gamma X\right)$, $\mathscr{D} \llbracket s_{1} \cup s_{2} \rrbracket \gamma X=\left(\mathscr{D} \llbracket s_{1} \rrbracket \gamma X\right) \cup\left(\mathscr{D} \llbracket s_{2} \rrbracket \gamma X\right)$.

d. $\mathscr{D} \llbracket \operatorname{new}(s) \rrbracket \gamma X=(\mathscr{D} \llbracket s \rrbracket \gamma\{\varepsilon\}) \| X$.

As usual, our main task is to relate $\mathcal{O}$ and $\mathscr{M}$. We shall prove

THeOREM 5.6. For all $t \in \mathscr{P P r o g}_{3}, \mathcal{O} \llbracket t \rrbracket=\mathscr{M} \llbracket t \rrbracket$.

The proof uses an auxiliary function $\mathscr{E}_{D}: \mathscr{P}_{a \imath} \rightarrow Q$ defined by

- $\mathscr{E}_{D} \llbracket \llbracket r_{1}, \ldots, r_{n} \rrbracket=\mathscr{E}_{D} \llbracket \llbracket r_{1} \rrbracket\|\ldots\| \mathscr{E}_{D} \llbracket \llbracket r_{n} \rrbracket$,

- $\quad \mathscr{E}_{D} \llbracket E \rrbracket=\{\varepsilon\}, \mathscr{E}_{D} \llbracket s ; r \rrbracket=\mathscr{D} \llbracket s \rrbracket \gamma_{D}\left(\mathscr{E}_{D} \llbracket r \rrbracket\right)$

We shall show that the following holds:

Claim

$$
\mathscr{E}_{D} \llbracket \varrho \rrbracket=\left\{\begin{array}{l}
\{\varepsilon\}, \text { if } \varrho=E, E, \ldots, E \\
\bigcup\left\{a \cdot \mathscr{E}_{D} \llbracket \varrho^{\prime} \rrbracket \mid \varrho \stackrel{a}{\rightarrow}_{D} \varrho^{\prime}\right\}, \text { otherwise. }
\end{array}\right.
$$

Once this claim has been established, we are done: By the usual argument, it implies that $\mathscr{E}_{D} \llbracket \varrho \rrbracket=\mathcal{O}_{D} \llbracket \varrho \rrbracket$; hence, in particular,

$$
\mathcal{O} \llbracket\langle D \mid s\rangle \rrbracket=\mathcal{O}_{D} \llbracket\left[s ; E \rrbracket=\mathscr{E}_{D} \llbracket s ; E \rrbracket=\mathscr{D} \llbracket s \rrbracket \gamma_{D}\{\varepsilon\}=\mathscr{M} \llbracket\langle D \mid s\rangle \rrbracket .\right.
$$

The claim is proved by showing that $\mathscr{E}_{D}$ satisfies $\left({ }^{*}\right): \Psi\left(\mathscr{E}_{D}\right)=\mathscr{E}_{D}$, where $\Psi$ is defined, for each $\mathscr{F}_{D} \in \mathscr{P}_{a r} \rightarrow Q$, by

$$
\Psi\left(\mathscr{F}_{D}\right)(\varrho)=\left\{\begin{array}{l}
\{\varepsilon\}, \text { if } \varrho=E, \ldots, E \\
\bigcup\left\{a \cdot \widetilde{\mathscr{F}} \llbracket \llbracket \varrho^{\prime} \rrbracket \mid \varrho \stackrel{a}{\rightarrow}_{D} \varrho^{\prime}\right\}, \text { otherwise. }
\end{array}\right.
$$

We prove $\left(^{*}\right)$ by induction on the complexity of $\varrho=r_{1}, \ldots, r_{m}$, which we define as the entity $\langle k, c(\varrho)\rangle$, where $k \geqq 0$ is the number of unguarded occurrences of some $x_{j}(1 \leqq j \leqq n)$ in some $r_{i}(1 \leqq i \leqq m)$. Moreover, $c(\varrho)$ is defined as $c\left(r_{1}\right)+\ldots+c\left(r_{m}\right)$, where $c(E)=0, \quad c(s ; r)=c(s)+c(r), \quad$ and $\quad c(a)=c(x)=1$, $c\left(s_{1} ; s_{2}\right)=c\left(s_{1} \cup s_{2}\right)=1+c\left(s_{1}\right)+c\left(s_{2}\right), c($ new $(s))=1+c(s)$. (We recall here that $x$ does occur unguarded in, e.g., new $(a) ; x ; E$.) We order the entities $\langle k, c\rangle$ by putting $\langle k, c\rangle\left\langle\left\langle k^{\prime}, c^{\prime}\right\rangle\right.$ whenever $k\left\langle k^{\prime}\right.$ or $k=k^{\prime}$ and $c<c^{\prime}$.

Stage 1 . We first consider the case that complexity $(\varrho)=\langle 0, \ldots\rangle$. If $\varrho=r, \varrho^{\prime}$ we show that $\Phi\left(\mathscr{E}_{D}\right)\left(r, \varrho^{\prime}\right)=\mathscr{E}_{D} \llbracket r, \varrho^{\prime} \rrbracket$ by an argument similar to that in section 3 , stage 1 of the proof of theorem 3.9. Here we use, in addition, that, if

$$
\frac{\varrho_{1} \stackrel{a_{D}}{\varrho_{D}}}{\varrho_{2} \stackrel{a_{D}}{\rightarrow_{D}}}
$$


then complexity $\left(\varrho_{1}\right)<$ complexity $\left(\varrho_{2}\right)$. If $\varrho=r$, we distinguish various subcases. If $r=E$, the claim is obvious. If $r=s ; r^{\prime}$, we argue by case analysis on the structure of $s$. We discuss two typical subcases:

- $s \equiv s_{1} ; s_{2}$. Then

$$
\begin{aligned}
& \Phi\left(\mathscr{E}_{D}\right)\left(\left(s_{1} ; s_{2}\right) ; r\right)= \\
& \bigcup\left\{a \cdot \mathscr{E}_{D} \llbracket \llbracket \tilde{\varrho} \rrbracket \mid\left(s_{1} ; s_{2}\right) ; r \stackrel{a}{\rightarrow}_{D} \tilde{\varrho}\right\}=\left(\text { def. } T_{3}\right) \\
& \bigcup\left\{a \cdot \mathscr{E}_{D} \llbracket \tilde{\varrho} \rrbracket \mid s_{1} ;\left(s_{2} ; r\right) \stackrel{a}{\rightarrow}{ }_{D} \tilde{\varrho}\right\}=
\end{aligned}
$$

(since $c\left(s_{1} ;\left(s_{2} ; r\right)\right)<c\left(\left(s_{1} ; s_{2}\right) ; r\right)$, we may apply the ind. hyp.)

$\mathscr{E}_{D} \llbracket s_{1} ;\left(s_{2} ; r\right) \rrbracket=\left(\right.$ def. $\left.\mathscr{E}_{D}, \mathscr{D}\right)$

$\mathscr{E}_{D} \llbracket\left(s_{1} ; s_{2}\right) ; r \rrbracket$.

- $s \equiv \operatorname{mew}\left(s^{\prime}\right)$.

$\Phi\left(\mathscr{E}_{D}\right)($ new $(s) ; r)=$

$\bigcup\left\{a \cdot \mathscr{E}_{D} \llbracket \tilde{\varrho} \rrbracket \mid(\right.$ new $(s) ; r \stackrel{a}{\rightarrow} D \tilde{\varrho}\}=\left(\right.$ def. $\left.T_{3}\right)$

$\bigcup\left\{a \cdot \mathscr{E}_{D} \llbracket \tilde{\varrho} \rrbracket \mid r, s ; E \stackrel{a}{\rightarrow}_{D} \tilde{\varrho}\right\}=$

(since $c(r, s ; E)<c($ new $(s) ; r)$, we may apply the ind. hyp.)

$\mathscr{E}_{D} \llbracket r, s ; E \rrbracket=\left(\right.$ def. $\left.\mathscr{E}_{D}\right)$

$\mathscr{E}_{D} \llbracket r \rrbracket \| \mathscr{E}_{D} \llbracket s ; E \rrbracket=\left(\right.$ def. $\left.\mathscr{E}_{D}, \mathscr{D}\right)$

$\left(\mathscr{D} \llbracket s \rrbracket \gamma_{D}\{\varepsilon\}\right) \| \mathscr{E}_{D} \llbracket r \rrbracket=$

$\mathscr{D} \llbracket$ new $(s) \rrbracket \gamma_{D}\left(\mathscr{E}_{D} \llbracket r \rrbracket\right)=$

$\mathscr{E}_{D} \llbracket$ new $(s) ; r \rrbracket$.

Stage $k+1$. Assume that $\left({ }^{*}\right)$ holds for any $\varrho$ with at most $k$ unguarded occurrences of some $x_{i}$. Now consider a $\varrho$ with $k+1$ unguarded occurrences. All cases are as before, but for the case $\varrho=r, r=s ; r^{\prime}, s=x_{i}$, for some $x_{i} \in \mathscr{X}$. Then

$$
\begin{aligned}
& \Phi\left(\mathscr{E}_{D}\right)\left(x_{i} ; r^{\prime}\right)= \\
& \bigcup\left\{a \cdot \mathscr{E}_{D} \llbracket \tilde{\varrho} \rrbracket \mid x_{i} ; r^{\prime} \stackrel{a}{\rightarrow}_{D} \tilde{\varrho}\right\}=\left(\text { def. } T_{3}\right) \\
& \bigcup\left\{a \cdot \mathscr{E}_{D} \llbracket \tilde{\varrho} \rrbracket \mid g_{i} ; r^{\prime} \stackrel{a}{\rightarrow}_{\nu} \varrho\left\{\left(\text { with } x_{i} \Leftarrow g_{i} \text { in } D\right)=\right.\right.
\end{aligned}
$$

(since $g_{i}$ is guarded, we may apply stage $k$ )

$\mathscr{E}_{D}\left[g_{i} ; r^{\prime}\right]=$

$\mathscr{E}_{D} \llbracket x_{i} ; r^{\prime} \rrbracket$, where the last equality holds by the definition of $\mathscr{D}$.

We conclude this section with two

\section{REMARKS.}

1. It has been shown by IJ. J. Aalbersberg and P. America (personal communication) that the expressive powers of $\|$ and of new (...) are incomparable: There exists $t_{1} \in \mathscr{P P r o g}_{1}$ such that for no $t_{3} \in \mathscr{P}_{\text {rog }}$, $\mathcal{O} \llbracket t_{1} \rrbracket=\mathcal{O} \llbracket t_{3} \rrbracket$, and vice versa.

2. In [1], process creation is also considered in a nonuniform setting, in the sense of, e.g., the language of the next section. 


\section{Communication with value passing.}

We conclude our list of four specimen languages analyzed with metric tools with a discussion of a nonuniform language $\mathscr{L}_{4}$ which is best seen as an extension of $\mathscr{L}_{2}$ from section 4 . The atomic actions of $\mathscr{L}_{4}$ are no longer uninterpreted symbols $a$ from some alphabet $A$, but, instead, assignments $v:=e$, for $v$ an individual variable and $e$ an expression, and communication actions $c$ ? $v$ or $c ! e$. Also, booleans $b$ are introduced appearing as tests in conditional statements. Accordingly, the semantic models now incorporate states, i.e. mappings from individual variables $v$ to elements $\alpha$ in some set $V$ of values.

We first collect some syntactic preparations. We introduce the set $(v \in)$ Indv of individual variables and $(c \in) C$ of channels. Channel names $c$ appear in the communication actions $c ? v$ and $c ! e$. Synchronization of two such actions is defined similarly to that of $c, \bar{c}$ in section 4 . In addition, however, at the moment of successful synchronization the assignment $v:=e$ takes place. Assuming that $c ? v$ occurs in some component $s_{1}$, and $c ! e$ in a component $s_{2}$ of the parallel statement $s_{1} \| s_{2}$, the current value of $e$ is transmitted by the sender $s_{2}$ over the channel $c$ to the receiver $s_{1}$, where it is (instantaneously) assigned to the variable $v$. Furthermore, we introduce the syntactic classes $(e \in) \mathscr{E} x p$ of expressions and $(b \in) \mathscr{B}$ ool of booleans. For simplicity, we assume some elementary syntax for $\mathscr{E} x p$ and $\mathscr{B} o o l$, and leave this unspecified here. We only postulate that no complications such as side-effects or nontermination arise in the evaluation of some $e$ or $b$.

We now give

Definition 6.1. Let $\mathscr{X}=\left\{x_{1}, \ldots, x_{n}\right\}$ be as before.

a. $\left(s \in \mathscr{L}_{4}\right)$.

$s::=v:=e|c ? v| c ! e|x|$ if $b$ then $s_{1}$ else $s_{2} f i\left|s_{1} ; s_{2}\right| s_{1}|| s_{2}$, with $x \in \mathscr{X}$.

b. $\left(g \in \mathscr{L}_{4}^{g}\right) . g::=v:=e|c ? v| c ! e|g ; s|$ if $b$ then $g_{1}$ else $g_{2} f i \mid g_{1} \| g_{2}$.

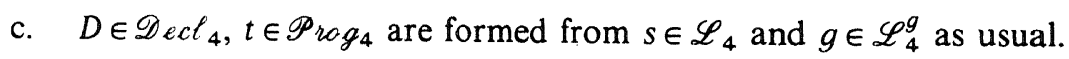

Remark. For simplicity, $\mathscr{L}_{4}$ has no form of nondeterminism.

Some semantic preparations are contained in

\section{Definition 6.2.}

a. $(\alpha \in) V$ is the set of values, $\{t t, f f)$ is the set of truth-values.

b. $(\sigma \in) \Sigma=\operatorname{Ind} v \rightarrow V$ is the set of states.

c. $(\eta \in) H=\Sigma \cup \Delta$, where

$(\delta \in) \Delta=\{c ? v \mid c \in C, v \in \operatorname{In} d v\} \cup\{c ! \alpha \mid c \in C, \alpha \in V\}$. 
d. For $e \in \mathscr{E} x \not h, \llbracket e \rrbracket(\sigma)$ denotes its value in state $\sigma$; for $b \in \mathscr{B}$ cot, $\llbracket b \rrbracket(\sigma)$ denotes its truth-value in state $\sigma$.

\section{REMARKS.}

1. The reader may always take $\mathbb{Z}$ for $V$ to give some realistic flavour to our considerations.

2. The set $H$ serves technical purposes in the definitions below. For given input $\sigma$, computations yield elements $\eta \in H$ as output. These may be distinguished into "normal" $\eta \in \Sigma$ and "abnormal" $\eta \in \Delta$, where the latter results from onesided (and therefore failing) attempts at synchronization $c ? v$ or $c !$ e.

We proceed with the definition of the transition system $T_{4}$. This time, transitions

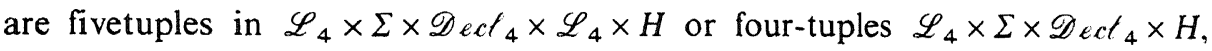
written as

$$
\begin{aligned}
& \langle s, \sigma\rangle \rightarrow_{D}\left\langle s^{\prime}, \eta\right\rangle, \\
& \langle s, \sigma\rangle \rightarrow_{D} \eta,
\end{aligned}
$$

respectively. $T_{4}$ is defined, applying a self-explanatory style of abbreviating rules, in

Definition 6.3.

$$
\begin{gathered}
\langle v:=e, \sigma\rangle \rightarrow_{D} \sigma\{\alpha / v\}, \text { where } \alpha=\llbracket e \rrbracket(\sigma) \\
\left\{\begin{array}{l}
\langle c ? v, \sigma\rangle \rightarrow_{D} c ? v \\
\langle c ! e, \sigma\rangle \rightarrow_{D} c ! \alpha
\end{array}, \text { where } \alpha=\llbracket e \rrbracket(\sigma)\right. \\
\frac{\left\langle s_{i}, \sigma\right\rangle \rightarrow_{D}\left\langle s^{\prime}, \eta\right\rangle \mid \eta}{\left\langle\text { if } b \text { then } s_{1} \text { else } s_{2} f i, \sigma\right\rangle \rightarrow_{D}\left\langle s^{\prime}, \eta\right\rangle \mid \eta}
\end{gathered}
$$

where $s_{i}=s_{1}\left(s_{2}\right)$ in case $\llbracket b \rrbracket(\sigma)=t t(f f)$

$$
\begin{gathered}
\frac{\langle s, \sigma\rangle \rightarrow_{D}\left\langle s^{\prime}, \eta\right\rangle \mid \eta}{\langle s ; \tilde{s}, \sigma\rangle \rightarrow_{D}\left\langle s^{\prime} ; \tilde{s}, \eta\right\rangle \mid\langle\tilde{s}, \eta\rangle} \\
\frac{\langle s, \sigma\rangle \rightarrow_{D}\left\langle s^{\prime}, \eta\right\rangle \mid \eta}{\langle s|| \tilde{s}, \sigma\rangle \rightarrow_{D}\left\langle s^{\prime} \mid \tilde{s}, \eta\right\rangle \mid\langle\tilde{s}, \eta\rangle} \\
\langle\tilde{s}|| s, \sigma\rangle \rightarrow_{D}\left\langle\tilde{s} \mid s^{\prime}, \eta\right\rangle \mid\langle\tilde{s}, \eta\rangle \\
\frac{\left\langle s_{1}, \sigma\right\rangle \rightarrow_{D}\left\langle s^{\prime}, c ? v\right\rangle,\left\langle s_{2}, \sigma\right\rangle \rightarrow_{D}\left\langle s^{\prime \prime}, c ! \alpha\right\rangle}{\left\langle s_{1}|| s_{2}, \sigma\right\rangle \rightarrow_{D}\left\langle s^{\prime}|| s^{\prime \prime}, \sigma\{\alpha / v\}\right\rangle}
\end{gathered}
$$

and the three obvious variations in case $s^{\prime}, s^{\prime \prime}$ or both are missing 


$$
\frac{\langle g, \sigma\rangle \rightarrow_{D}\left\langle s^{\prime}, \eta\right\rangle \mid \eta}{\langle x, \sigma\rangle \rightarrow_{D}\left\langle s^{\prime}, \eta\right\rangle \mid \eta}, \text { with } x \in g \text { in } D
$$

Before we define $\mathcal{C} \llbracket t \rrbracket$ and $\mathcal{O}_{D} \llbracket s \rrbracket$ we first introduce the process domain $P$ as solution of

$$
P \cong\left\{p_{0}\right\} \cup\left(\Sigma \rightarrow \mathscr{P}_{\text {compact }}\left(H \times i d_{1 / 2}(P)\right)\right)
$$

with the discrete metric on $\Sigma$ and $H$.

\section{REMARKS.}

1. In (6.1), $\mathscr{P}_{\text {compact }}(\cdot)$ denotes all compact subsets of $(\cdot)$. This is necessitated by the fact that the "alphabet" $H$ is, in general, infinite (contrary to the finite $A$ in section 4).

2. We leave for another occasion discussion of the equation

$$
P^{\prime} \cong\{\varepsilon\} \cup\left(\Sigma \rightarrow \mathscr{P}_{\text {compact }}\left(H \cdot i d_{1 / 2}\left(P^{\prime}\right)\right)\right)
$$

determining $P^{\prime}$ as possible "linear time" alternative for $P$. This discussion will in particular have to clarify the role of '. ' versus ' $x$ ' in a nonuniform context.

The operational semantics are given in

Definition 6.4 (operational semantics for $\mathscr{P}_{\text {rog }} g_{4}, \mathscr{L}_{4}$ ).

a. $\mathcal{O}: \mathscr{P r o g}_{4} \rightarrow P$ is given by $\mathcal{O} \llbracket\langle D \mid s\rangle \rrbracket=\mathcal{O}_{D} \llbracket s \rrbracket$.

b. $\mathcal{O}_{D}: \mathscr{L}_{4} \rightarrow P$ is given by:

$\mathcal{O}_{D} \llbracket s \rrbracket=\lambda \sigma .\left(\left\{\left\langle\sigma^{\prime}, \mathcal{O}_{D} \llbracket s^{\prime} \rrbracket\right\rangle\left|\langle s, \sigma\rangle \rightarrow_{D}\left\langle s^{\prime}, \sigma^{\prime}\right\rangle\right\rangle \cup\left\{\left\langle\sigma^{\prime}, p_{0}\right\rangle \mid\langle s, \sigma\rangle \rightarrow_{D} \sigma^{\prime}\right\}\right) ;\right.$

where the transitions are with respect to $T_{4}$.

Remark. Just as in definition $4.3, \mathcal{O}_{D}$ does not take into account transitions stemming from failing communications, signalled here by the format $\langle s, \sigma\rangle \rightarrow_{D}\left\langle s^{\prime}, \delta\right\rangle \mid \delta$ with $\delta \in \Delta$.

For $P$ as in (6.1), we can define the usual operators $\cup, \circ, \|$. We restrict ourselves to the definition of $\|$, here involving the auxiliary operators $\mathbb{L}$ and $\mid$.

Definition 6.5. Let $P$ be as in (6.1), and let $(X \in) \mathscr{P}$ abbreviate $\mathscr{P}_{\text {compact }}\left(H \times i d_{1 / 2}(P)\right)$. We define the operator $\|$ as fixed point of $\Phi_{\| l}:(P \times P \rightarrow P) \rightarrow$ $\rightarrow(P \times P \rightarrow P)$, where, for $\phi \in P \times P \rightarrow P$ and $\phi$ ndi, $\Phi_{\| \mid}(\phi)=d f \cdot \tilde{\phi}_{\|}$is given by 


$$
\tilde{\phi}_{||}(p)(q)=\left\{\begin{array}{l}
p, \text { if } q=p_{0} \\
q, \text { if } p=p_{0} \\
\lambda \sigma .\left(\hat{\phi}_{\mathbb{L}}(p(\sigma))(q) \cup \hat{\phi}_{\mathbb{L}}(q(\sigma))(p) \cup \hat{\phi}_{\mid, \sigma}(p(\sigma))(q(\sigma))\right)
\end{array}\right.
$$

where $\hat{\phi}_{\mathbb{L}}: \mathscr{P} \times P \rightarrow \mathscr{P}$ is defined by

$$
\hat{\phi}_{\mathbb{L}}(X)(q)=\left\{\left\langle\eta, \phi\left(p^{\prime}\right)(q)\right\rangle \mid\left\langle\eta, p^{\prime}\right\rangle \in X\right\},
$$

and $\hat{\phi}_{\mid, \sigma}: \mathscr{P} \times \mathscr{P} \rightarrow \mathscr{P}$ is defined by

$$
\hat{\phi}_{\mid, \sigma}(X)(Y)=\left\{\left\langle\sigma\{\alpha / v\}, \phi\left(p^{\prime}\right)\left(q^{\prime}\right)\right\rangle \mid\left\langle c ? v, p^{\prime}\right\rangle \in X,\left\langle c ! \alpha, q^{\prime}\right\rangle \in Y \text { or vice versa }\right\} .
$$

We are now ready for the defintion of $\mathscr{M} \llbracket t \rrbracket$ and $\mathscr{D} \llbracket t \rrbracket$. Let $(\gamma \in) \Gamma_{4}=\mathscr{X} \rightarrow P$, let $\mathscr{M}: \mathscr{P}_{\text {rog }_{4}} \rightarrow P$ and $\mathscr{D}: \mathscr{L}_{4} \rightarrow\left(\Gamma_{4} \rightarrow P\right)$. We give

Definition 6.6 (denotational semantics for $\mathscr{L}_{4}, \mathscr{P}_{\text {reg }}$ ).

a. $\quad \mathscr{M} \llbracket\langle D \mid s\rangle \rrbracket=\mathscr{D} \llbracket s \rrbracket \gamma_{D}$.

b. $\gamma_{D}$ is as usual.

c. $\mathscr{D} \llbracket v:=e \rrbracket \gamma=\lambda \sigma .\left\{\left\langle\sigma\{\alpha / v\}, p_{0}\right\rangle\right\}$, with $\alpha=\llbracket e \rrbracket(\sigma), \mathscr{D} \llbracket c ? v \rrbracket \gamma=\lambda \sigma .\left\{\left\langle c ? v, p_{0}\right\rangle\right\}$, $\mathscr{D} \llbracket c ! e \rrbracket \gamma=\lambda \sigma .\left\{\left\langle c ! \alpha, p_{0}\right\rangle\right\}$, with $\alpha=\llbracket e \rrbracket(\sigma)$,

$\mathscr{D} \llbracket$ if $b$ then $s_{1}$ else $s_{2}$ fi $\rrbracket=\lambda \sigma . i f \llbracket b \rrbracket(\sigma)=$ tt then $\mathscr{D} \llbracket s_{1} \rrbracket \gamma \sigma$ else $\mathscr{D} \llbracket s_{2} \rrbracket \gamma \sigma f i$, and

$\mathscr{D} \llbracket x \rrbracket \gamma, \mathscr{D} \llbracket s_{1}$ op $s_{2} \rrbracket \gamma$ for op $=; \cup, \|$, as usual.

One last step is necessary before we can formulate our final result. We define the abstraction mapping abs: $P \rightarrow P^{\prime \prime}$, where $P^{\prime \prime}$ satisfies

$$
P^{\prime \prime} \cong\left\{p_{0}\right\} \cup\left(\Sigma \rightarrow \mathscr{P}_{\text {compact }}\left(\Sigma \times i d_{1 / 2}\left(P^{\prime \prime}\right)\right)\right)
$$

by putting abs $=$ fixed point $\left(\Psi_{a b s}\right)$, with $\Psi_{a b s}:\left(P \rightarrow P^{\prime \prime}\right) \rightarrow\left(P \rightarrow P^{\prime \prime}\right)$ defined by: For $\psi \in P \rightarrow P^{\prime \prime}, \Psi_{a b s}(\psi)={ }^{d s} \cdot \tilde{\psi}_{a b s}$ is given by

and, for $p \neq p_{0}$,

$$
\tilde{\psi}_{a b s}\left(p_{0}\right)=p_{0},
$$

$$
\tilde{\psi}_{a b s}(p)=\lambda \sigma \cdot \hat{\psi}(p(\sigma))
$$

and

$$
\hat{\psi}(X)=\left\{\left\langle\sigma, \psi\left(p^{\prime}\right)\right\rangle \mid\left\langle\sigma, p^{\prime}\right\rangle \in X\right\} .
$$

Note that the last clause deletes pairs $\left\langle\delta, p^{\prime}\right\rangle$ from $X$.

We finally have:

TheOREM 6.7. For each $t \in \mathscr{P}_{\mathrm{rog}_{4},}, \mathcal{O} \llbracket t \rrbracket=($ abs $\circ \mathscr{M}) \llbracket t \rrbracket$.

Proof. By the usual contractivity argument. 


\section{Acknowledgements.}

The work would not have been possible without the essential contributions of the Amsterdam concurrency group and its affiliates. We acknowledge in particular the work of Joost Kok and Jan Rutten who first realized the crucial role of contractivity arguments in comparing concurrency semantics. Pierre America, Ernst-Rüdiger Olderog and Jeff Zucker have worked together with us on the papers [10], [1], both of which were instrumental for the present paper.

\section{REFERENCES}

1. P. America and J. W. de Bakker, Designing equivalent semantic models for process creation, in Proc. Advanced School on Mathematical Models for the Semantics of Parallelism (M. Venturini Zilli, ed.), LNCS 280, Springer, 1987, pp. 21-80 (to appear in Theoretical Computer Science).

2. P. America, J. W. de Bakker, J. N. Kok and J. J. M. M. Rutten, Operational semantics of a parallel object-oriented language, 13th ACM Symposium on Principles of Programming Languages, St. Petersburg, Florida, January 13-15, 1986, pp. 194-208.

3. P. America, J. W. de Bakker, J. N. Kok and J. J. M. M. Rutten, Denotational semantics of a parallel object-oriented language, Report CS-R8626, Centre for Mathematics and Computer Science, Amsterdam, 1986. To appear in Information and Computation.

4. P. America and J. J. M. M. Rutten, Solving reflexive domain equations in a category of complete metric spaces, in Proc. of the Third Workschop on Mathematical Foundations of Programming Language Semantics (M. Main, A. Melton, M. Mislove and D. Schmidt, eds.), LNCS 298, Springer, 1988, pp. 254-288.

5. K. Apt and G. Plotkin, Countable nondeterminism and random assignment, JACM 33(4), (1986), 724-767.

6. J. W. de Bakker, J. A. Bergstra, J. W. Klop, and J.-J. Ch. Meyer, Linear time and branching time semantics for recursion with merge, TCS 34 (1984), 135-156.

7. J. W. de Bakker, J. N. Kok, J.-J. Ch. Meyer, E.-R. Olderog, and J. I. Zucker, Contrasting themes in the semantics of imperative concurrency, in Current Trends in Concurrency: Overviews and Tutorials (J. W. de Bakker, W. P. de Roever, G. Rozenberg, eds.), LNCS 224, Springer (1986), $51-121$.

8. J. W. de Bakker and J.-J. Ch. Meyer, Order and metric in the stream semantics of elemental concurrency, Acta Informatica 24 (1987), 491-511.

9. J. W. de Bakker, J.-J. Ch. Meyer, and E.-R. Olderog, Infinite streams and finite observations in the semantics of uniform concurrency, TCS 49 (1987), 87-112.

10. J. W. de Bakker, J.-J. Ch. Meyer, E.-R. Olderog, and J. I. Zucker, Transition systems, metric spaces and ready sets in the semantics of uniform concurrency, Journal of Comp. Syst. Sci. 36 (1988), 158-224.

11. J. W. de Bakker and J. I. Zucker, Processes and the denotational semantics of concurrency, Inform. and Control 54 (1982), 70-120.

12. J. W. de Bakker and J. I. Zucker, Processes and a fair semantics for the ADA rendez-vous, in: Proc. 10th ICALP (J. Diaz ed.), LNCS 154, Springer (1983), 52-66.

13. J. A. Bergstra and J. W. Klop, A convergence theorem in process algebra, Report CS-R8733, Centre for Mathematics and Computer Science, Amsterdam, 1987.

14. J. Dugundji, Topology, Allen and Bacon, Rockleigh, N.J. 1966.

15. R. Engelking, General topology, Polish Scientific Publishers 1977.

16. M. Hennessy and G. D. Plotkin, Full abstraction for a simple parallel programming language, in: Proceedings 8th MFCS (J. Becvar ed.), LNCS 74 Springer (1979), 108-120. 
17. C. A. R. Hoare, Communicating Sequential Processes, Prentice-Hall Int., Englewood Cliffs, New Jersey, 1985.

18. J. N. Kok and J. J. M. M. Rutten, Contractions in comparing concurrency semantics, Report CS R8755, Centre for Mathematics and Computer Science, Amsterdam, 1987, to appear in Proc. ICALP 1988

19. J.-J. Ch. Meyer, Merging regular processes by means of fixed point theory, TCS 45 (1986), $193-$ 260.

20. J.-J. Ch. Meyer and E.-R. Olderog, Hiding in stream semantics of uniform concurrency, Report IR-125, Free University, Amsterdam, 1987.

21. J.-J. Ch. Meyer, and E. P. de Vink, Applications of compactness in the Smyth powerdomain of streams, in: Proc. TAPSOFT '87 (H. Ehrig, R. Kowalski, G. Levi, U. Montanari, eds.), LNCS 249, Springer (1987), 241-255.

22. R. Milner, A calculus for communicating systems, LNCS 92, Springer, 1980.

23. M. Nivat, Infinite words, infinite trees, infinite computations, in: Foundations of Computer Science III.2 (J. W. de Bakker, J. van Leeuwen, eds.), Mathematical Centre Tracts 109, Amsterdam (1979), 3-52

24. G. D. Plotkin, A powerdomain construction, SIAM Journal of Computing, Vol. 5, No. 3 (1976), 452-487.

25. G. D. Plotkin, A structural approach to operational semantics, Report DAIMI FN-19, Comp. Sci. Dept., Aarhus Univ., 1981.

26. G. D. Plotkin, An operational semantics for CSP, in: D. Bjørner (ed.): Formal Description of Programming Concepts II, North-Holland (1983), 199-223.

27. M. B. Smyth, Quasi uniformities, reconciling domains with metric spaces, in Proceedings of the 3rd Workshop on Mathematical Foundations of Programming Language Semantics (M. Main, A. Melton, M. Mislove and D. Schmidt, eds.), LNCS 298, Springer, 1988, pp. 236-253. 